\title{
Temperatura, umidade relativa e atraso na instalação da atmosfera controlada no armazenamento de maçã 'Fuji'
}

\author{
Temperature, relative humidity and delay in installation of controlled atmosphere storage of the \\ 'Fuji' apple
}

\author{
Auri Brackmann ${ }^{1 *}$ Rogério de Oliveira Anese ${ }^{\mathrm{II}}$ Josuel Alfredo Vilela Pinto \\ Cristiano André Steffens ${ }^{\mathrm{II}}$ Affonso José Wietzke Guarienti ${ }^{\mathrm{II}}$
}

RESUMO

O objetivo deste trabalho foi avaliar o atraso na instalação da atmosfera controlada, da exposição à temperatura mais elevada $\left(3^{\circ} \mathrm{C}\right)$ e do uso de baixa umidade relativa no início do armazenamento sobre a qualidade da maçã da cultivar 'Fuji'. O delineamento experimental utilizado foi o inteiramente casualizado, com 10 tratamentos e quatro repetições. Os tratamentos avaliados consistiram de combinações de retardo na instalação da AC, por meio do $A R$, uso da alta temperatura $\left(3^{\circ} \mathrm{C}\right)$ e baixa umidade relativa (85\%), por um período de um mês e posterior armazenamento em AC, com $1,0 \mathrm{kPa}$ de $\mathrm{O}_{2}+<0,5 \mathrm{kPa}$ de $\mathrm{CO}_{2}$, na temperatura de $0,5^{\circ} \mathrm{C}$ (UR 96\%), do uso da AC em duas temperaturas $\left(0,5^{\circ} \mathrm{C}\right.$ e $\left.-0,5^{\circ} \mathrm{C}\right)$ e também pela exposição ao alto $\mathrm{CO}_{2}$ no período inicial de armazenamento. Pelos resultados, o uso da baixa umidade associada ou não à temperatura de $3^{\circ} \mathrm{C}$ no primeiro mês de armazenamento reduziu a incidência de degenerescência e podridão e manteve a firmeza da polpa. A temperatura de $0,5^{\circ} \mathrm{C}$ foi eficiente na redução da incidência de degenerescência, podridões e manutenção da firmeza, quando comparada à temperatura de $0,5^{\circ} \mathrm{C}$. O atraso da instalação da atmosfera controlada combinado com a temperatura de $3^{\circ} \mathrm{C}$ no período inicial de armazenamento não é recomendado, pois causa elevada incidência de podridões e baixa firmeza da polpa. A alta pressão parcial de $\mathrm{CO}_{2}$ ou a baixa pressão parcial de $\mathrm{O}_{2}$ causa maior incidência de degenerescência da polpa.

Palavras-chave: Malus domestica, armazenamento, póscolheita.

\section{ABSTRACT}

The aim of this research was to evaluate the delay in installation of a controlled atmosphere, exposure to highe temperature $\left(3^{\circ} \mathrm{C}\right)$ at the beginning of the storage period and use of low humidity also at the beginning of storage on the quality of apple 'Fuji'. The experimental design was a completely randomized with ten treatments and four replicates. The treatments evaluated were combinations of delay in installation of the $\mathrm{CA}$, through $\mathrm{RH}$, high temperature $\left(3^{\circ} \mathrm{C}\right)$ usage and low humidity (85\%) for a period of one month and later storage with $\mathrm{AC} 1.0 \mathrm{kPa}$ of $\mathrm{O}_{2}+<0.5 \mathrm{kPa}$ of $\mathrm{CO}_{2}$ in temperature of $-0.5^{\circ} \mathrm{C}$ ( $R H$ 96\%), use of $A C$ in two temperatures ( 0.5 and $-0.5^{\circ} \mathrm{C}$ ), and also by exposure to high $\mathrm{CO}_{2}$ in the initial period of storage. The results showed that the use of low humidity associated or not to the temperature of $3^{\circ} \mathrm{C}$ in the first month of storage reduced the incidence of breakdown, decay and maintained the firmness of flesh. The temperature of $0.5^{\circ} \mathrm{C}$ is effective in reducing the incidence of breakdown, decay and maintenance of firmness when compared to the temperature of $0.5^{\circ} \mathrm{C}$. The delay of the controlled installation atmosphere combined with a temperature of $3^{\circ} \mathrm{C}$ in the initial period of storage is not recommended because it causes high incidence of decay and low pulp firmness. The high partial pressure of $\mathrm{CO}_{2}$ or low $\mathrm{O}_{2}$ partial pressure of relevant causes incidence of breakdown.

Key words: Malus domestica, storage, postharvest.

\section{INTRODUÇÃO}

A maçã 'Fuji' apresenta excelente sabor, polpa crocante e suculenta, o que propicia boa aceitação no mercado consumidor brasileiro e também no exterior. Em razão disso, o Rio Grande do Sul apresentou, na safra 2007-08, uma produção da maçã 'Fuji' de aproximadamente 139 mil toneladas,

IDepartamento de Fitotecnia, Universidade Federal de Santa Maria (UFSM), Centro de Ciências Rurais (CCR). Av. Roraima, nº 1000, Cidade Universitária, Bairro Camobi, 97105-900, Santa Maria, RS, Brasil. E-mail: auribrackmann@gmail.com. *Autor para correspondência

IIDepartamento de Fitotecnia, Universidade do Estado de Santa Catarina (UDESC), Lages, SC, Brasil.

IIISoloeste Indústria e Comércio Importação e Exportação Ltda, Iomere, SC, Brasil. 
representado cerca de 35\% do total de maçã produzida pelo Estado (AGAPOMI, 2009). Devido ao curto período de colheita, ocorre uma oferta de produto muito superior à capacidade de absorção do mercado. Assim, as empresas utilizam o armazenamento em atmosfera controlada (AC) para manter a qualidade dos frutos durante longos períodos, o que permite o abastecimento do mercado interno na entressafra. Nesse período, no entanto, ocorrem perdas principalmente decorrentes da degenerescência da polpa e podridão que, em determinados casos, atinge cerca de 50\% das maçãs armazenadas (BRACKMANN \& SAQUET, 1995). A degenerescência da polpa e as podridões são decorrentes das inadequadas condições de umidade relativa, temperatura, pressão parcial de $\mathrm{O}_{2}$ e $\mathrm{CO}_{2}$, em que os frutos são armazenados.

$\mathrm{O}$ armazenamento em AC combina baixas concentrações de oxigênio $\left(\mathrm{O}_{2}\right)$ e altas concentrações de gás carbônico $\left(\mathrm{CO}_{2}\right)$, juntamente com baixa temperatura e controle da umidade (BRACKMANN et al., 2005). Na maçã 'Fuji', as condições de ACinibem a biossíntese do etileno, fitohormônio responsável por desencadear os processos de amadurecimento (GORNY \& KADER, 1997), reduzem a respiração, mantêm as características físico-químicas e, em alguns casos, controlam a incidência de podridões (KE et al., 1991). Com relação à temperatura, à medida que essa diminui, também ocorre redução na velocidade das reações bioquímicas no fruto, as quais são responsáveis pela maturação e senescência, que causam perda de qualidade. A temperatura não somente interfere nos processos metabólicos do fruto, como também modifica o efeito de outros fatores da atmosfera da câmara, como a umidade relativa (CHITARRA \& CHITARRA, 2005). A umidade relativa (UR) afeta a transpiração, altera a atividade respiratória e produção de etileno (BENDER, 1986), além de quando muito elevada, causa degenerescência de polpa e propicia condições adequadas para o desenvolvimento de patógenos causadores de podridões (LUNARDI, 2003).

Durante o armazenamento, devido ao processo respiratório, há aumento no nível de $\mathrm{CO}_{2}$ dentro da câmara, o que pode causar grandes perdas por degenerescência de polpa em maçã 'Fuji'. A degenerescência é um distúrbio fisiológico que se caracteriza por um escurecimento generalizado da polpa do fruto, que adquire aspecto farináceo, podendo ainda apresentar pequenas cavidades, com a formação de tecido de cortiça na polpa (MAZARO et al., 1994). Na maçã 'Fuji', a degenerescência ocorre, principalmente, devido à difícil difusibilidade do $\mathrm{CO}_{2}$ do interior da polpa do fruto para a atmosfera externa, principalmente nas semanas iniciais de estocagem, em que a suscetibilidade à degenerescência é maior (BRACKMANN et al., 1999), sendo a rápida instalação da AC um fator responsável por esse aumento (WATKINS et al., 1997). Em vista disso, uma opção para contornar tal problema seria o atraso na instalação das condições de AC (ARGENTA et al., 2000; BORTOLUZZI-MAAG \& BRACKMANN, 2001). As empresas que estocam maçãs 'Fuji' fazem o uso da cal para reduzir o $\mathrm{CO}_{2}$ a um nível bem baixo $(<0,8 \%)$, o que eleva os custos e ainda ocupa espaço dentro da câmara. Associada ao $\mathrm{CO}_{2}$, a baixa temperatura também pode acarretar danos à célula do fruto, por alterar a permeabilidade das membranas celulares (FLORESCANTILLANO \& GIRARDI, 2004). Alguns autores sugerem o resfriamento gradativo como forma de minimizar essa injúria, visto que o estresse por temperatura é maior no início do armazenamento (LITTLE \& BARRAND, 1989; FLORES-CANTILLANO \& GIRARDI, 2004).

Diante disso, o objetivo do presente trabalho foi avaliar o atraso na instalação da atmosfera controlada, da exposição à temperatura mais elevada $\left(3^{\circ} \mathrm{C}\right)$, do uso de baixa umidade relativa, da exposição a um alto nível de $\mathrm{CO}_{2}$ no início do período de armazenamento, bem como do efeito da temperatura e do nível de $\mathrm{O}_{2}$ sobre a qualidade físico-química da maçã da cultivar 'Fuji' durante o armazenamento em AC.

\section{MATERIAL E MÉTODOS}

O experimento foi conduzido no Núcleo de Pesquisa em Pós-colheita (NPP) do Departamento de Fitotecnia da Universidade Federal de Santa Maria, com maçãs da cultivar 'Fuji' provenientes de um pomar comercial de São Joaquim, Santa Catarina (SC). Após a colheita, os frutos foram transportados até o NPP, onde então foram selecionados, eliminando aqueles com lesões, defeitos e baixo calibre. Em seguida, as amostras experimentais foram homogeneizadas. Na instalação do experimento, os frutos apresentavam os seguintes parâmetros de maturação: índice de iodo-amido: 7,06; firmeza de polpa: $70,51 \mathrm{~N}$; sólidos solúveis totais: 12,13 ${ }^{\circ}$ Brix; e acidez: $3,62 \mathrm{meq} 100 \mathrm{~mL}^{-1}$. O experimento foi constituído no delineamento inteiramente casualizado, com 10 tratamentos e quatro repetições, em que cada unidade experimental foi composta de 20 frutos.

As condições de armazenamento avaliadas foram: (1) $1,0 \mathrm{kPa} \mathrm{O}_{2}+<0,5 \mathrm{kPa} \mathrm{CO}_{2}$ na temperatura de $0,5^{\circ} \mathrm{C}$; (2) $1,0 \mathrm{kPaO}_{2}+<0,5 \mathrm{kPa} \mathrm{CO}_{2}$ na temperatura de $0,5^{\circ} \mathrm{C}$; (3) $0,8 \mathrm{kPaO}_{2}+<0,5 \mathrm{kPa} \mathrm{CO}_{2}$ na temperatura de $0,5^{\circ} \mathrm{C}$; (4) $1,0 \mathrm{kPa} \mathrm{O}_{2}+<0,5 \mathrm{kPa} \mathrm{CO}_{2}$, primeiro mês na temperatura de $3^{\circ} \mathrm{C}$ em $\mathrm{AC}$ e o restante do período a - 
$0,5^{\circ} \mathrm{Cem} \mathrm{AC;} \mathrm{(5)} 1,0 \mathrm{kPaO}_{2}+<0,5 \mathrm{kPaCO}_{2}$, primeiro mês na temperatura de $3^{\circ} \mathrm{C}$ em $\mathrm{AR}$ e o restante do período a $-0,5^{\circ} \mathrm{C}$ em AC; (6) $1,0 \mathrm{kPa} \mathrm{O}+<0,5 \mathrm{kPa} \mathrm{CO}_{2}$, primeiro mês em baixa UR (85\%) a $-0,5^{\circ} \mathrm{C}$ em $\mathrm{AC}$ e o restante do período em alta UR (96\%) na temperatura de $-0,5^{\circ} \mathrm{C}$; (7) $1,0 \mathrm{kPa} \mathrm{O}+<0,5 \mathrm{kPa} \mathrm{CO}_{2}$, primeiro mês em baixa UR (85\%) a $3^{\circ} \mathrm{C}$ em AC e o restante do período em alta UR (96\%) na temperatura de $-0,5^{\circ} \mathrm{C}$; (8) $1,0 \mathrm{kPa} \mathrm{O}_{2}+<0,5 \mathrm{kPa}$ $\mathrm{CO}_{2}$, primeiro mês em baixa UR (85\%) a $3^{\circ} \mathrm{C}$ em AR e o restante do período em alta UR (96\%) na temperatura de $-0,5^{\circ} \mathrm{C}$ em AC; (9) $1,0 \mathrm{kPa} \mathrm{O}+2,0 \mathrm{kPa} \mathrm{CO}_{2}$ na temperatura de $-0,5^{\circ} \mathrm{C}$; (10) $1,0 \mathrm{kPa} \mathrm{O}_{2}+2,0 \mathrm{kPa} \mathrm{CO}_{2}$ nos três meses iniciais e o restante do período com $<0,5 \mathrm{kPa}$ $\mathrm{CO}_{2}$ à $-0,5^{\circ} \mathrm{C}$.

Os frutos foram acondicionados em caixas plásticas com capacidade para 20,0kg e então armazenados em minicâmaras experimentais de AC, com volume de $0,232 \mathrm{~m}^{3}$, que permaneceram no interior de câmaras frigoríficas de $45 \mathrm{~m}^{3}$. No interior das minicâmaras dos tratamentos com baixa umidade, a UR foi mantida em $85 \%$ ( $\pm 3 \%$ ) no primeiro mês e em $96 \%$ $( \pm 1 \%)$ no restante do período. Nos demais tratamentos, a UR também foi mantida em $96 \%( \pm 1 \%)$. Para a manutenção da baixa UR no primeiro mês, foi utilizada uma bomba de membrana, que circula o ar do interior da minicâmara para dentro de um recipiente contendo sílica gel, a qual era substituída após sua saturação. A umidade relativa foi monitorada semanalmente, por meio de um psicrômetro. A temperatura das câmaras frigoríficas foi controlada automaticamente por termostatos de alta precisão $\left( \pm 0,1^{\circ} \mathrm{C}\right)$, sendo monitorada diariamente por meio de termômetros de mercúrio introduzidos na polpa de frutos. A instalação e o controle das condições de AC foram realizados conforme BRACKMANN et al. (2005).

Depois de transcorridos sete meses de armazenamento, os frutos foram retirados das minicâmaras, e cada amostra foi dividida em duas subamostras, uma para análise na saída da câmara e outra para análise aos sete dias de exposição a $20^{\circ} \mathrm{C}$, simulando o período de comercialização. Os parâmetros analisados foram: firmeza de polpa, com auxílio de um penetrômetro com ponteira de 11,0mm; acidez titulável, pela titulação de $10,0 \mathrm{~mL}$ de suco com $\mathrm{NaOH} 0,1 \mathrm{~N}$; sólidos solúveis totais (SST), medido com um refratômetro; incidência de podridões, pela observação e contagem dos frutos que apresentavam podridões; degenerescência, pela visualização e contagem dos frutos que apresentavam sintomas; e coloração, com um colorímetro.

Para cada variável avaliada, foi efetuada análise de variância, sendo as médias comparadas pelo teste de Scott-knott, com 5\% de probabilidade de erro.
As variáveis expressas em porcentagem foram transformadas pela fórmula arc.sen $\sqrt{\mathrm{x} / 100}$, antes da análise de variância.

\section{RESULTADOS E DISCUSSÃO}

Após sete meses de armazenamento, o teor de SST e a acidez titulável na saída da câmara e aos sete dias de exposição a $20^{\circ} \mathrm{C}$ não diferiram, estatisticamente, entre os tratamentos (dados não apresentados), confirmando resultados de trabalhos anteriores (BRACKMANN et al., 1998).

O tratamento em que os frutos foram armazenados na temperatura de $-0,5^{\circ} \mathrm{C}(\mathrm{T} 2)$ apresentou menores perdas da firmeza da polpa, menor incidência de podridões e degenerescência, quando comparado ao tratamento com temperatura de $0,5^{\circ} \mathrm{C}(\mathrm{T} 1)$, tanto na avaliação realizada na saída da câmara, quanto após sete dias na temperatura de $20^{\circ} \mathrm{C}$ (Tabelas 1 e 2). Entretanto, não houve diferença estatística para o parâmetro degenerescência na análise na saída da câmara e podridão na análise na saída da câmara e aos sete dias a $20^{\circ} \mathrm{C}$. Provalvemente, na temperatura de $0,5^{\circ} \mathrm{C}$, houve menor produção e acúmulo de $\mathrm{CO}_{2}$ nos tecidos, já que a degenerescência é atribuída ao dano causado pelo acúmulo desse gás nos tecidos (CASTRO et al., 2008). Em trabalho anterior, BRACKMANN et al. (1998) também encontraram menor incidência de degenerescência com a redução da temperatura de $0,5^{\circ} \mathrm{C}$ para $-0,5^{\circ} \mathrm{C}$.

Com relação à concentração parcial de $\mathrm{O}_{2}$ durante a armazenagem, na avaliação realizada na saída da câmara, não houve diferença nos parâmetros analisados entre as condições testadas (Tabela 1). Já após sete dias de exposição a $20^{\circ} \mathrm{C}$, os frutos que foram submetidos a $1,0 \mathrm{kPa}$ de $\mathrm{O}_{2}$ (T2) apresentaram menor incidência de degenerescência em comparação com os frutos submetidos a $0,8 \mathrm{kPa}$ (T3) (Tabela 2). CERETTA (2003) também encontrou maior incidência de degenerescência em maçãs 'Gala' armazenadas sob baixas pressões parciais de $\mathrm{O}_{2}$ no início do período de estocagem. Durante o armazenamento, a baixa concentração de $\mathrm{O}_{2}$ pode induzir a produção de energia pela rota anaeróbica, podendo ocorrer a formação de alcoóis e aldeído acético, o que causa sabor desagradável e danos aos frutos (GIL et al., 1998), podendo apresentar polpa marrom de aspecto úmido (CERETTA, 2003).

A alta temperatura no primeiro mês $\left(3^{\circ} \mathrm{C}\right)$ em AC e o posterior armazenamento sob temperatura de $0,5^{\circ} \mathrm{C}$ (T4), na análise na saída da câmara, causaram alta incidência de podridões e maior perda de firmeza da polpa, em comparação com a temperatura de $-0,5^{\circ} \mathrm{C}$ 
Tabela 1 - Qualidade da maçã 'Fuji’ em função da temperatura, das condições de atmosfera controlada e da umidade relativa após sete meses de armazenamento. Santa Maria - RS, 2004.

\begin{tabular}{|c|c|c|c|c|c|}
\hline Tratamento & Temperatura & Firmeza da polpa & Podridão & Degenerescência & Cor \\
\hline $\mathrm{O}_{2}+\mathrm{CO}_{2}(\mathrm{kPa})$ & $\left({ }^{\circ} \mathrm{C}\right)$ & $(\mathrm{N})$ & $(\%)$ & $(\%)$ & Ângulo Hue \\
\hline $1,0+<0,5$ & 0,5 & $53,4 b^{I}$ & $7,00 \mathrm{~b}$ & $10,0 \mathrm{a}$ & $101,4 \mathrm{a}$ \\
\hline $1,0+<0,5$ & $-0,5$ & 55,9 a & $3,00 \mathrm{~b}$ & $7,88 \mathrm{a}$ & 103,5 a \\
\hline $0,8+<0,5$ & $-0,5$ & 56,0 a & $4,00 \mathrm{~b}$ & 8,00 a & 100,7 a \\
\hline $1,0+<0,5$ & $-0,5^{\mathrm{II}}$ & $53,4 \mathrm{~b}$ & $22,0 \mathrm{a}$ & $6,50 \mathrm{a}$ & $102,4 \mathrm{a}$ \\
\hline $1,0+<0,5$ & $-0,5^{\mathrm{III}}$ & $51,6 \mathrm{~b}$ & 20,3 a & $6,00 \mathrm{a}$ & 100,6 a \\
\hline $1,0+<0,5$ & $-0,5^{\mathrm{IV}}$ & 57,6 a & $1,00 \mathrm{~b}$ & $3,00 \mathrm{a}$ & 98,7 b \\
\hline $1,0+<0,5$ & $-0,5^{\mathrm{V}}$ & 55,4 a & $6,00 \mathrm{~b}$ & $7,00 \mathrm{a}$ & 96,5 b \\
\hline $1,0+<0,5$ & $-0,5^{\mathrm{VI}}$ & $51,2 \mathrm{~b}$ & $20,6 \mathrm{a}$ & $5,50 \mathrm{a}$ & 98,8 b \\
\hline $1,0+2,0$ & $-0,5$ & 59,0 a & 11,9 a & 8,42 a & 102,5 a \\
\hline $1,0+<0,5$ & $-0,5^{\mathrm{VII}}$ & $54,2 \mathrm{~b}$ & $23,1 \mathrm{a}$ & 9,56 a & $99,0 \mathrm{~b}$ \\
\hline CV (\%) & & 5,19 & 39,9 & 26,0 & 2,6 \\
\hline
\end{tabular}

${ }^{\mathrm{I}}$ Médias não seguidas pela mesma letra diferem estatisticamente entre si pelo teste de Scott-knott, em nível de 5\% de probabilidade de erro.

${ }^{\text {II }}$ Primeiro mês na temperatura de $3^{\circ} \mathrm{C}$ em $\mathrm{AC}$.

${ }^{\text {III }}$ Primeiro mês na temperatura de $3^{\circ} \mathrm{C}$ em AR.

${ }^{\text {IV }}$ Primeiro mês em baixa umidade relativa (85\%)

${ }^{v}$ Primeiro mês em baixa umidade relativa (85\%) a $3^{\circ} \mathrm{C}$, restante do período a $96 \%$ de UR.

${ }^{\mathrm{VI}}$ Primeiro mês em baixa umidade relativa (85\%) a $3^{\circ} \mathrm{C}$ em $\mathrm{AR}$, restante do período a $96 \%$ de UR.

${ }^{\mathrm{VII}} 2 \mathrm{kPa}$ de $\mathrm{CO}_{2}$ nos três meses iniciais.

durante todo o período de estocagem (T2) (Tabela 1). O mesmo resultado foi observado quando se utilizaram a alta temperatura e o atraso em um mês na instalação da AC (T5). Provavelmente, a temperatura mais elevada e o atraso na instalação da AC por um mês contribuíram para a aceleração do metabolismo e a consequente degradação das paredes celulares, ocasionando menor firmeza e menor resistência ao ataque de patógenos causadores de podridões. Esses resultados contrariam os reportados por BRACKMANN et al. (2000), os quais afirmam que a alta temperatura $\left(2,5^{\circ} \mathrm{C}\right)$ durante os 40 dias iniciais em AC reduziu a incidência de podridões em maçãs 'Fuji'. No entanto, aos sete dias de exposição a $20^{\circ} \mathrm{C}$, a alta temperatura no mês inicial manteve a firmeza da polpa mais elevada quando armazenada em AC; porém, quando submetida ao atraso na instalação da AC por um mês (T5), houve menor retenção da firmeza da polpa (Tabela 2). Os demais parâmetros: podridão, degenerescência e cor não apresentaram diferença estatística entre as condições com alta temperatura $\left(3^{\circ} \mathrm{C}\right)$ em $\mathrm{AC}$ no primeiro mês (T4) e alta temperatura em AR no primeiro mês (T5) com a testemunha (T2).

Quanto à baixa umidade relativa no primeiro mês (T6, T7 e T8), na avaliação realizada na saída da câmara, a cor da epiderme dos frutos se apresentou mais amarela em relação à testemunha (T2) (Tabela 1), provavelmente devido ao estresse hídrico ocasionado pela perda de umidade, que pode ter aumentado a síntese de etileno e este aumentado a degradação da clorofila, pois BENDER (1986) e NAKANO et al. (2003) afirmam que o estresse hídrico aumenta a síntese de etileno em maçãs e caquis, respectivamente. A baixa umidade relativa, associada ou não com a temperatura de $3^{\circ} \mathrm{C}$ no primeiro mês (T6 e T7), proporcionou menor perda da firmeza da polpa e menor incidência de podridões; no entanto, não apresentou diferença estatística da testemunha (T2). A menor perda da firmeza pode ser decorrente da desidratação causada pela baixa umidade (MAZARO et al., 1994), e a menor incidência de podridão pode estar relacionada à baixa umidade, a qual causa uma condição adversa à proliferação de fungos, pois, de acordo com SCHWARZ (1994), a elevada incidência de podridões está ligada à alta umidade relativa durante o armazenamento. O tratamento com baixa umidade relativa na temperatura de $3^{\circ} \mathrm{C}$, em AR, no mês inicial e posterior armazenamento em AC (T8), ocasionou alta incidência de podridão e maior perda da firmeza (Tabela 1). Certamente, o efeito do atraso na instalação da AC contribuiu para o aumento da incidência de podridão e diminuiu a firmeza da polpa pelo fato de ter havido maior metabolismo degradativo no período inicial de armazenagem. $\mathrm{Na}$ análise aos sete dias a $20^{\circ} \mathrm{C}$, o comportamento dos frutos para os parâmetros analisados foi semelhante aos resultados obtidos na análise na saída da câmara, porém a incidência de podridão não apresentou diferença estatística da testemunha (Tabela 2). De modo 
Tabela 2 - Qualidade da maçã 'Fuji’ em função da temperatura, das condições de atmosfera controlada e da umidade relativa após sete meses de armazenamento mais sete dias de exposição à temperatura de $20^{\circ} \mathrm{C}$. Santa Maria - RS, 2004

\begin{tabular}{|c|c|c|c|c|c|}
\hline Tratamento & Temperatura & Firmeza da polpa & Podridão & Degenerescência & Cor \\
\hline $\mathrm{O}_{2}+\mathrm{CO}_{2}(\mathrm{kPa})$ & $\left({ }^{\circ} \mathrm{C}\right)$ & $(\mathrm{N})$ & $(\%)$ & $(\%)$ & Ângulo Hue \\
\hline $1,0+<0,5$ & 0,5 & $54,4 b^{I}$ & 58,5 a & $24,1 \mathrm{a}$ & 91,9 a \\
\hline $1,0+<0,5$ & $-0,5$ & $55,4 \mathrm{a}$ & 33,8 a & $16,3 \mathrm{~b}$ & 98,3 a \\
\hline $0,8+<0,5$ & $-0,5$ & 56,9 a & 33,8 a & $25,2 \mathrm{a}$ & 92,5 a \\
\hline $1,0+<0,5$ & $-0,5^{\mathrm{II}}$ & $56,1 \mathrm{a}$ & $31,9 \mathrm{a}$ & $18,8 \mathrm{~b}$ & $95,1 \mathrm{a}$ \\
\hline $1,0+<0,5$ & $-0,5^{\mathrm{III}}$ & $51,8 \mathrm{~b}$ & $38,5 \mathrm{a}$ & $15,4 \mathrm{~b}$ & $93,1 \mathrm{a}$ \\
\hline $1,0+<0,5$ & $-0,5^{\mathrm{IV}}$ & 56,3 a & 30,3 a & $19,0 \mathrm{~b}$ & $92,8 \mathrm{a}$ \\
\hline $1,0+<0,5$ & $-0,5^{\mathrm{V}}$ & $58,6 \mathrm{a}$ & $21,8 \mathrm{a}$ & $16,1 \mathrm{~b}$ & $95,3 \mathrm{a}$ \\
\hline $1,0+<0,5$ & $-0,5^{\mathrm{VI}}$ & $52,8 \mathrm{~b}$ & 32,9 a & $10,4 \mathrm{~b}$ & 95,6 a \\
\hline $1,0+2,0$ & $-0,5$ & 59,0 a & 30,9 a & 30,6 a & 95,2 a \\
\hline $1,0+<0,5$ & $-0,5^{\mathrm{VII}}$ & $56,3 \mathrm{a}$ & 53,6 a & $30,8 \mathrm{a}$ & 94,6 a \\
\hline CV (\%) & & 3,46 & 28,1 & 24,2 & 2,74 \\
\hline
\end{tabular}

${ }^{\text {I }}$ Médias não seguidas pela mesma letra diferem estatisticamente entre si pelo teste de Scott-knott, em nível de 5\% de probabilidade de erro.

${ }^{\text {II }}$ Primeiro mês na temperatura de $3^{\circ} \mathrm{C}$ em $\mathrm{AC}$.

${ }^{\text {III }}$ Primeiro mês na temperatura de $3^{\circ} \mathrm{C}$ em $\mathrm{AR}$.

IV Primeiro mês em baixa umidade relativa (85\%)

${ }^{\mathrm{V}}$ Primeiro mês em baixa umidade relativa (85\%) a $3^{\circ} \mathrm{C}$, restante do período a $96 \%$ de UR.

${ }^{\mathrm{VI}}$ Primeiro mês em baixa umidade relativa (85\%) a $3^{\circ} \mathrm{C}$ em $\mathrm{AR}$, restante do período a $96 \%$ de UR.

VII 2 kPa de $\mathrm{CO}_{2}$ nos três meses iniciais.

geral, a baixa umidade relativa causou baixa incidência de degenerescência, estando de acordo com resultados encontrados por LITTLE \& BARRAND (1989).

A condição com alto $\mathrm{CO}_{2}$ durante o período de estocagem (T9), na análise na saída da câmara, manteve a firmeza da polpa; no entanto, não houve diferença estatística da condição com baixo $\mathrm{CO}_{2}$ (T2). $\mathrm{O}$ alto $\mathrm{CO}_{2}$ nos três meses iniciais (T10) causou maior perda da firmeza da polpa, alta incidência de podridões e apresentou coloração mais amarela em relação à testemunha (T2). Aos sete dias de exposição a $20^{\circ} \mathrm{C}$, o uso do alto $\mathrm{CO}_{2}$, tanto durante todo o período, quanto nos três primeiros meses (T10), causou maior incidência de degenerescência (Tabela 2). Esses resultados confirmam os reportados por BRACKMANN et al. (1998 e 1999), em que a sensibilidade da maçã 'Fuji' ao alto $\mathrm{CO}_{2}$ e as condições de atmosfera controlada são maiores no início do período de armazenamento.

\section{CONCLUSÕES}

Para o armazenamento de maçã 'Fuji', a temperatura de $-0,5^{\circ} \mathrm{C}$ e a baixa umidade relativa associada ou não à temperatura de $3^{\circ} \mathrm{C}$ são mais eficientes, pois reduzem a incidência de degenerescência e podridões e mantêm a firmeza da polpa.

O atraso da instalação da atmosfera controlada por um mês eleva a incidência de podridões e diminui a firmeza da polpa. A alta pressão parcial de $\mathrm{CO}_{2}(2,0 \mathrm{kPa})$ ou a baixa pressão parcial de $\mathrm{O}_{2}(0,8 \mathrm{kPa})$ causam maior incidência de degenerescência da polpa.

\section{REFERÊNCIAS}

AGAPOMI - Associação Gaúcha dos Produtores de Maças. Disponível em: <http://www.agapomi.com.br/arquivos/Res.safra.pdf>. Acesso em 17 jun.2009.

ARGENTA, L.C. et al. Delaying establishment of controlled atmosphere or $\mathrm{CO}_{2}$ exposure reduces 'Fuji' apple $\mathrm{CO}_{2}$ injury without excessive fruit quality loss. Postharvest Biology and Technology, Amsterdam, v.20, p.221-229, 2000. Disponível em: <http://dx.doi.org/10.1016/S0925-5214(00)00134-4>. Acesso em 15 dez.2008. doi: 10.1016/S0925-5214(00)00134-4.

BENDER, J.R. Colheita e armazenagem. In: EMPASC. Manual da cultura da macieira. Florianópolis: EMPASC, 1986. p.521-562.

BORTOLUZZI-MAAG, G.; BRACKMANN, A. Qualidade da maçã 'Fuji' relacionada com a umidade relativa e a temperatura de armazenamento. Revista Científica Rural, Bagé, v.6, n.1, p.01-05, 2001.

BRACKMANN, A. et al. Frigoconservação de maçã 'Fuji' em duas temperaturas e em atmosfera controlada. Revista Brasileira de Agrociência, Pelotas, v.4, n.1, p.26-30, 1998. Disponível em: <http://www.ufpel.tche.br/faem/agrociencia/ v4n1/artigo05.pdf>. Acesso em 23 fev.2008.

BRACKMANN, A. et al. Controle da degenerescência da polpa da maçã Fuji com concentrações dinâmicas de $\mathrm{O}_{2}$ e $\mathrm{CO}_{2}$ e redução da umidade relativa durante o armazenamento em atmosfera 
controlada. Revista Ciência Rural, Santa Maria, v.29, n.3, p.459-463, 1999. Disponível em: <http://www.scielo.br/pdf/ cr/v29n3/a13v29n3.pdf $>$. Acesso em 12 ago.2008. doi: 10.1590/S0103-84781999000300013.

BRACKMANN, A. et al. Condições de atmosfera controlada, temperatura e umidade relativa no armazenamento de maçã 'Fuji'. Ciência e Agrotecnologia, Lavras, v.29, n.4, p.803809, 2005. Disponível em: <http://www.scielo.br/pdf/cagro/ v29n4/a12v29n4.pdf $>$. Acesso em 25 set.2008.

BRACKMANN, A. et al. Conservação da maçã 'Fuji' sob diferentes temperaturas, umidades relativas e momentos de instalação da atmosfera de armazenamento. Ciência Rural, Santa Maria, v.30, n.1, p.81-84, 2000. Disponível em: <http:/ /www.scielo.br/pdf/cr/v30n1/a13v30n1.pdf >. Acesso em 13 out.2008. doi: 10.1590/S0103-84782000000100013.

BRACKMANN, A.; SAQUET, A.A. Efeito das condições de atmosfera controlada sobre a ocorrência de degenerescência em maçãs 'Fuji'. Scientia Agricola, Piracicaba, v.52, n.2, p.263-267, 1995. Disponível em: <http://www.scielo.br/pdf/ sa/v52n2/10.pdf $>$. Acesso em 18 out.2008. doi: 10.1590/ S0103-90161995000200010.

CASTRO, E. et al. Biochemical factors associated with a $\mathrm{CO}_{2}-$ induced flesh browning disorder of Pink Lady apples. Postharvest Biology and Technology, Califórnia, v.48, p.182-191, 2008.

CERETTA, M. Tolerância de maçãs 'Gala' e 'Fuji' a baixas temperaturas e extremas pressões parciais de $\mathrm{O}_{2}$ e $\mathrm{CO}_{2}$ durante o armazenamento em atmosfera controlada. 2003. 70f. Tese (Doutorado em Agronomia) - Curso de Pósgraduação em Agronomia, Universidade Federal de Pelotas.

CHITARRA, M.I.F.; CHITARRA, A.B. Pós-colheita de frutas e hortaliças: fisiologia e manuseio. Lavras: UFLA, 2005. V1.

FLORES-CANTILlANO, F.; GIRARDI, C.L. Maçã póscolheita: distúrbios fisiológicos. Bento Gonçalves: Empresa brasileira de pesquisa agropecuária, 2004. 109p.

GORNY, G.R.; KADER, A.A. Low oxygen and elevated carbon dioxide atmospheres inhibit ethylene biosynthesis in preclimacteric and climacteric apple fruit. Journal American Society for Horticultural Science, Alexandria, v.122, n.4, p. 542-546, 1997.

GIL, M.I. et al. Responses of 'Fuji' apples to ascorbic acid treatments and low oxygen atmosphere. HortScience, Alexandria, v.33, n.2, p.305-309, 1998.

KE, D. et al. Physiology and prediction of fruit tolerance to low-oxygen atmosphere. Journal of the American Society for Horticultural Science, Alexandria, v.116, n.2 p.253260, 1991.

LITTLE, C.R.; BARRAND, L. The effect of preharvest, postharvest and storage conditions on some fruit disorders. In: INTERNATIONAL CONTROLLED ATMOSPHERE RESEARCH CONFERENCE, 5, 1989, Washington . Proceedings... Washington: Washington Satate University, 1989. V.1, p.185-192.

LUNARDI, R. et al. Suculência e solubilização de pectinas em maçãs 'Gala', armazenadas em atmosfera controlada, em dois níveis de umidade relativa. Ciência Rural, Santa Maria, v.34, n.3, p.743-747, 2004. Disponível em: <http://www.scielo.br/ pdf/cr/v34n3/a14v34n3.pdf $>$. Acesso em 22 nov.2008. doi: 10.1590/S0103-84782004000300014.

MAZARO, S.M. et al. Condições de atmosfera controlada para o armazenamento de maçã 'Fuji'. In: CONGRESSO BRASILEIRO DE FRUTICULTURA, 13., 1994, Salvador, Ba. Anais... Salvador, Ba: Sociedade Brasileira de Fruticultura, 1994. V.2. 760p.

NAKANO, R. et al. Ethylene biosynthesis in detached young persimmon is initiated in calix and modulated by water loss from the fruit. Plant Physiology, Rockville, v.131, p.276286, 2003.

SCHWARZ, A. Relative humidity in cool stores: measurement conrtrol and influence of discret factors. Acta Horticulturae, Wageningen, n.368, p.867-892, 1994.

WATKINS, C.B. et al. A comparison of two carbon dioxiderelated injuries of apple fruit. In: INTERNATIONAL CONTROLLED ATMOSPHERE RESEARCH CONFERENCE, 7, 1997, Davis, EUA. Proceedings... Davis: University of California, 1997. V.2, 308p, p.119-124. 